\title{
NOTE ON THE GYROSCOPE
}

BY W. F. OSGOOD

By a gyroscope we mean a rigid body having an axis of material symmetry and free to turn about a certain fixed point $O$ of the axis. Let the gyroscope be acted on by any forces, and let it be required to determine the motion of the axis under the action of these forces. By far and away the most interesting and important case is that in which the component couple tending to produce rotation about the axis is nil, so that the component angular velocity about the axis is constant; denote it by $\nu$. Under this restriction the resultant of the applied forces can be expressed as a single force, $\mathfrak{F}$, acting at a point $P$ on the axis at unit distance from $O$, normally to $O P$, and by the force the peg at $O$ exerts.

Let $(5$ be the curve $P$ describes on the unit sphere about $O$. The problem is solved by the intrinsic equations:*

$$
A \frac{d v}{d t}=T, \quad A \kappa v^{2}+C \nu v=Q,
$$

where $C$ and $A$ are the moments of inertia about the axis and about a normal to the axis through $O$; $T$ and $Q$ are the components of $\mathfrak{F}$ along the tangent and normal (in the tangent plane of the sphere) of $\mathbb{E}$; and $\kappa$ is the bending of the cone determined by $O$ and $\mathbb{E}$,- or the rate at which the plane through $O$ tangent to $\sqrt{5}$ is turning when the point of tangency describes $\mathbb{S}$ with unit velocity.

The object of the present note is to point out a simple interpretation of these equations in terms of the motion of a material particle carrying a charge of electricity and moving in the electro-magnetic field of force generated by the north pole of a magnet situated at $O$. Let the mass, $m$, of the particle be $A$, or $m=A$; and let the particle be constrained to lie on the unit sphere about $O$. Let

$$
Q_{1}=A \kappa v^{2}, \quad Q_{2}=C \nu v .
$$

* See the author's paper, On the gyroscope, Transactions of this Society, vol. 23 (1922), p. 240. 
Then it is easily shown that the particle without any electrical charge will describe the path $\mathbb{E}$ if acted on by the tangential force $T$ and the normal force $Q_{1}$; for the equations of motion then become

$$
m \frac{d v}{d t}=T, \quad m \kappa v^{2}=Q_{1} .
$$

Now apply a charge, $e$, of (positive or negative) electricity to $m$. The force which the field exerts on the charge will be proportional to the velocity, $v$, of the charged particle in its path, and in a direction normal to the path and to $O P$. By suitably choosing $e$ this force can be made to equal precisely the negative of $Q_{2}$, or $-C \nu v$. If, then, the force which acts on the mass $m$ is the same force $\mathfrak{F}$ that acts on the gyroscope at $P$, with its components $T$ along the tangent and

$$
Q=Q_{1}+Q_{2}
$$

along the normal, the electro-magnetic force of the field on the electrical charge, namely, $-C \nu v$, will precisely eliminate the term $Q_{2}$ in $Q$ and leave only $Q_{1}$ to act on $m$. $^{*}$

The result may be formulated in the following theorem.

THEOREM. When the gyroscope is acted on by a force $\mathfrak{F}$ at $P$ normal to the axis, the point $P$ moves exactly as a particle of mass $m=A$ acted on by the same force $\mathfrak{F}$ and constrained to lie on the unit sphere about $O$ would move if $m$ were carrying a suitable charge e of electricity and the motion of $m$ thus charged took place in an electro-magnetic field generated by the north pole of a magnet situated at $O$.

\section{HARVARD UNIVERSITY}

* I am indebted to my colleague, Professor Kemble, for the suggestion of a radial electro-magnetic field and a charge of electricity moving in that field, as a means of taking care of that part of the force at right angles to the path, which corresponds to the term $C \nu v$. 